physics/0110053

\title{
Nonresonant Effects in One- and Two-Photon Transitions
}

\author{
Ulrich D. Jentschura $^{a), b)}$ and Peter J. Mohr ${ }^{b}$ ) \\ a) Institute of Theoretical Physics, \\ Dresden University of Technology, 01062 Dresden, Germany \\ b) National Institute of Standards and Technology, \\ Mail Stop 8401, Gaithersburg, Maryland 20899-8401, USA
}

\begin{abstract}
We investigate nonresonant contributions to resonant Rayleigh scattering cross sections of atoms. The problematic nonresonant contributions set a limit to the accuracy to which atomic spectra determine energy levels. We discuss the offresonance effects in one-photon transitions. We also show that off-resonance contributions for the $1 \mathrm{~S}-2 \mathrm{~S}$ two-photon transition in atomic hydrogen are negligible at current and projected levels of experimental accuracy. The possibility of a differential measurement for the detection of off-resonance effects in one-photon transitions in atomic hydrogen is discussed.
\end{abstract}

PACS numbers 31.15.-p, 12.20.Ds

Keywords Calculations and mathematical techniques in atomic and molecular physics, quantum electrodynamics - specific calculations 


\section{Introduction}

Recently, the dramatic progress in laser-spectroscopic experiments in atomic hydrogen [1] has sparked interest in theoretical calculations at highly improved accuracy [2 [5]. This raises interesting questions regarding the relation of the resonance peak in the scattering cross section, which is observed in experiments, and the actual difference in the real parts of the energies of the two levels involved in the atomic transition!. In short, one may ask to which level of accuracy atomic spectra determine energy levels. Related questions are of prime importance for the determination of fundamental constants [6].

These issues are most easily dealt with if one assumes that the scattering process is described to a good approximation by a Kramers-Heisenberg formula [7]. For one-photon transitions, the excitation of the atom from the ground state by a laser photon and photon emission? is well described by the two diagrams in Fig. 1. For two-photon transitions, the situation is more involved. We consider here a process where two interactions with laser-photons are accompanied by the emission of two photons (see Fig. 2).

It has been pointed out as early as 1952 [9], that the experimental spectrum of atomic hydrogen does not reproduce the energy level differences precisely, and that nonresonant contributions to photon scattering shift the observed resonance peaks relative to the energy level differences by a frequency $\delta \omega$ which is of the order of

$$
\delta \omega \sim \alpha^{2}(Z \alpha)^{6} \frac{m c^{2}}{\hbar}
$$

where $m$ is the electron mass, $\alpha$ is the fine structure constant, $Z$ the nuclear charge number, and $c$ the speed of light; $\hbar=h /(2 \pi)$ is the natural unit of action where $h$ denotes Planck's constant. This shift $\delta \omega$ occurs for one-photon transitions where the atom returns to its ground state after the photon emission. Later, the interesting fact that resonance peaks do not necessarily determine atomic energy levels was discussed in Ref. [10]. We also refer to the related investigations [11 17]. In a recent theoretical calculation of higher-order radiative effects for hydrogenic $\mathrm{P}$ states [20], it has also been pointed out that an accurate analysis of the line shape is necessary at the level of the current theoretical uncertainty.

A priori, the detection of a nonresonant contribution of the order of (1) in a one-photon transition would require the determination of the peak of a hydrogenic transition frequency with an uncertainty of roughly $10^{-7}$ relative to the width of the transition frequency [the natural radiative decay rate $\Gamma$ of a typical bound state in atomic hydrogen is of the order of $\alpha(Z \alpha)^{4}$ in units of $m c^{2} / \hbar$, and we consider the quantity $\left.\delta \omega / \Gamma\right]$. In Sec. 3, we will consider a differential cross section, in which the nonresonant contribution could be observed more clearly.

This paper is organized as follows: In Sec. 2, we estimate nonresonant frequency shifts in both one- and two-photon transitions with special attention devoted to the $1 \mathrm{~S}-2 \mathrm{~S}$ two-photon transition in hydrogen (our estimates are also of relevance for other two-photon processes). We do not consider the effect of radiative vertex corrections to the photon absorption and emission on the line shape in this paper. In Sec. 3, we consider a differential cross section measurement in which nonresonant effects could be observed with optical transition frequencies in atomic hydrogen. Conclusions are left to Sec 4 .

\footnotetext{
${ }^{1}$ in constrast to the "real part", the "imaginary part" of the energy $-\mathrm{i} \Gamma / 2$ corresponds to the "radiative width" of the state which is related to the "radiative lifetime" $\tau$ according to $1 / \tau=\Gamma / \hbar$

${ }^{2}$ This process is an elastic scattering process (the atom returns to the ground state) that contributes to the Rayleigh scattering cross section. This is in contrast to inelastic scattering of highly energetic photons by free or "approximately free" electrons (Compton scattering) and inelastic scattering in the low-frequency domain (Raman scattering); see, e.g., the discussion on pp. 374-376 of [8].
} 


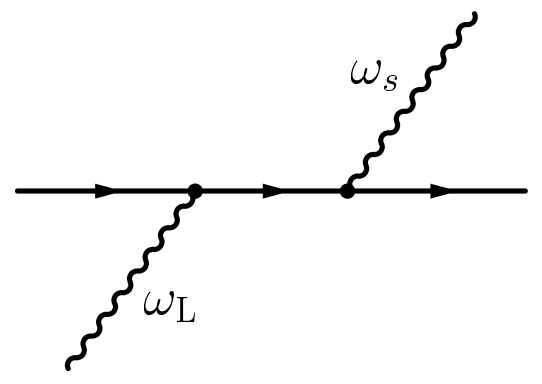

(a)

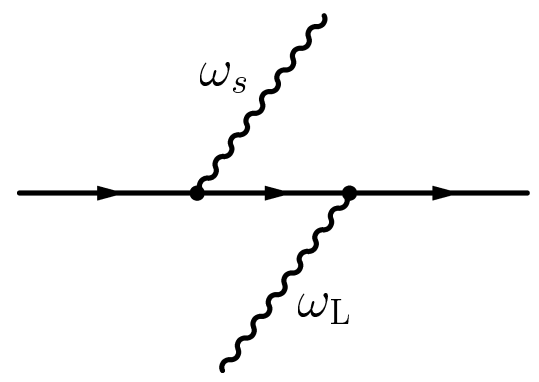

(b)

Figure 1: A two-photon process with absorption and emission. Time increases from left to right. The atom absorbs one laser photon with frequency $\omega_{\mathrm{L}}$ and emits one photon with frequency $\omega_{\mathrm{s}}$. The electron propagator is that of the bound electron, which is assumed to be in its ground state in both the initial and final configuration.

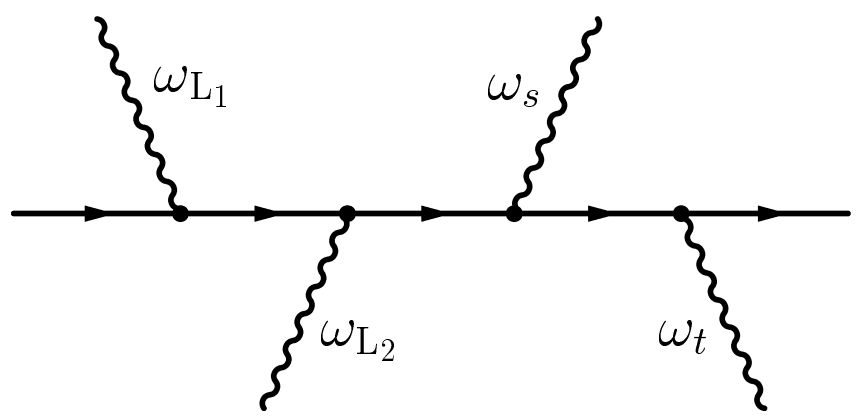

Figure 2: A four-photon process. Two laser photons with frequency $\omega_{\mathrm{L}_{1}}$ and $\omega_{\mathrm{L}_{2}}$ are absorbed, and two photons with frequencies $\omega_{s, t}$ are emitted. In many experiments, $\omega_{\mathrm{L}}=\omega_{\mathrm{L}_{1}}=\omega_{\mathrm{L}_{2}}$. As in Fig. 1, the cross section is given by the sum over permutations of the laser photons and the emitted photons. The resonance condition $2 \omega_{\mathrm{L}}=E_{2}-E_{1}$ corresponds to the absorption of two photons.

\section{One-- and Two-Photon Transitions: Off-Resonant Effects}

The first theoretical description of atomic two-photon transitions was given in Ref. [21], with the first experimental observation following in Ref. [22]. The description becomes less involved if one assumes that both the initial and the final state of the transition are "asymptotic states," i.e., states which can be used as initial and final states for $S$-matrix elements. This is a valid approximation only in the case of a long radiative lifetime for both the initial and the final state of the transition (of course, only the ground state will survive in the asymptotic limit of infinitely large time.)

We consider, within the "asymptotic-state" approximation, the differential cross section for a two-photon excitation process $|1\rangle \rightarrow|f\rangle$ through an intermediate state $|i\rangle$ (here, we assume the state $|1\rangle$ to be the ground state). We restrict the discussion to a situation in which two photons, one from each of two counter-propagating laser beams are absorbed by the atom. This eliminates first-order Doppler shifts $\delta \omega_{\mathrm{D}}= \pm \boldsymbol{k} \cdot \boldsymbol{v}$ and corresponds to the situation encountered in current 


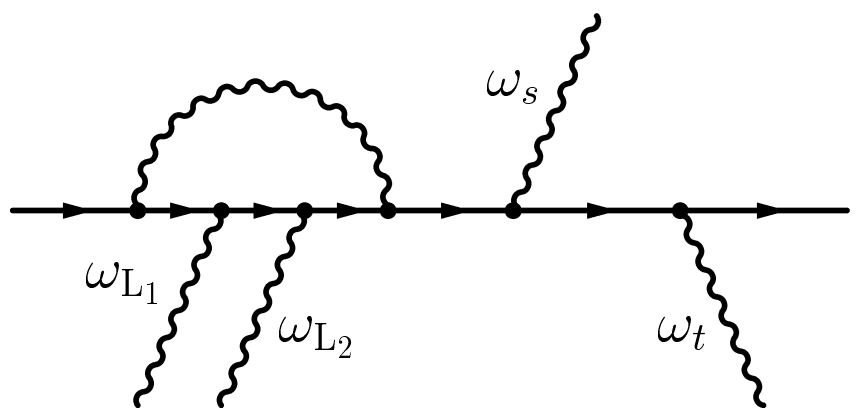

Figure 3: A four-photon process with a vertex correction. The vertex corrections are expected to give corrections of relative order $\alpha(Z \alpha)^{2}$ to the cross section.

high-precision experiments on $\mathrm{S} \rightarrow \mathrm{S}$ transitions in atomic hydrogen [1], where (first-order) Doppler-broadened background is suppressed. The cross section in this case is proportional to

$$
\left|\sum_{i} \frac{\left(\varepsilon_{\lambda} \cdot \boldsymbol{D}_{f i}\right)\left(\boldsymbol{\varepsilon}_{\lambda} \cdot \boldsymbol{D}_{i 1}\right)}{E_{i}-E_{1}-\hbar \omega_{\mathrm{L}}}\right|^{2} \delta\left(E_{f}-E_{i}-2 \hbar \omega_{\mathrm{L}}\right),
$$

where the sum over $i$ runs over all intermediate states, with the appropriate mutliplicity for degenerate states. The polarization vector of the laser beam is $\varepsilon_{\lambda} ; \omega_{\mathrm{L}}$ is the laser frequency and $\boldsymbol{D}_{i j}=\langle i|e \boldsymbol{x}| j\rangle$ is the dipole matrix element $[$.

As is evident from Eq. (2), for two-photon transitions, we can expect large resonant effects if the light of one of the lasers excites an intermediate resonance $\left(\hbar \omega_{\mathrm{L}} \approx E_{i}-E_{1}\right)$. In this case, the energy denominator in (2) becomes small (we would then have to include imaginary parts according to $\left.E_{i} \rightarrow E_{i}-\mathrm{i} \Gamma_{i} / 2\right)$ and the cross section peaks. Such an "intermediate-state" resonance occurs only if there exists an intermediate state with the appropriate energy.

For the current high-precision hydrogen measurements [1], all energy denominators in the summation in (2) are non-vanishing even at the peak of the two-photon resonance, which is near $\omega_{\mathrm{L}} \approx\left(E_{f}-E_{1}\right) /(2 \hbar)$. A possible exception would be the case of an atomic system in which there is accidentally an intermediate state present with an energy midway between $E_{1}$ and $E_{f}$, that is $E_{i} \approx\left(E_{f}-E_{1}\right) / 2$, but in general, this is not relevant to atomic hydrogen, because the spectrum is not equally spaced.

Although the Doppler-free two-photon $1 \mathrm{~S}-2 \mathrm{~S}$ resonance is extremely narrow, with a natural line width of $\Gamma_{f}=\Gamma_{2 S}$ (we recall that the natural width of the $2 \mathrm{~S}$ level in atomic hydrogen is $1.3 \mathrm{~Hz}$ [24]), nonresonant contributions need to be estimated in an improved framework that avoids the "asymptotic-state" approximation inherent in Eq. (2). To do this, we first consider a two-photon process with one photon being absorbed and one being emitted (see Fig. 1), as discussed by Low [9]. This approach has recently been applied in a study of the line shape in Ref. [17]. The atom is excited from its ground state $|1\rangle$ to an intermediate state $|i\rangle$ by interaction with a laser of frequency $\omega_{\mathrm{L}}$ and returns to the ground state $\left|1^{\prime}\right\rangle$ via spontaneous emission. The

\footnotetext{
${ }^{3}$ If Doppler-broadened background is not fully suppressed, then the Doppler-free cross section (2) manifests itself as a narrow peak which is superimposed on Doppler-broadened background. In this case, the Dopplerbroadened background must be taken into account [see Eq. (7.43) of [23]] via an additional additive contribution in (2) involving a factor $\exp \left[-\left(E_{f}-E_{1}-2 \hbar \omega_{\mathrm{L}}\right)^{2} /\left(2 k_{\mathrm{L}} v_{\mathrm{w}}\right)^{2}\right]$, where $v_{\mathrm{w}}=\sqrt{2 k_{\mathrm{B}} T / m}$ is the thermal velocity of the atoms (at the peak of the thermal probability distribution), and $k_{\mathrm{L}}=\omega_{\mathrm{L}} / c$.
} 
differential cross section is proportional to [0, 9]

$$
\frac{\mathrm{d} \sigma}{\mathrm{d} \Omega}\left(\varepsilon_{\mathrm{L}}, \boldsymbol{\varepsilon}_{\mathrm{S}}\right) \propto\left|\sum_{i} \frac{\left(\varepsilon_{\mathrm{S}}^{*} \cdot \boldsymbol{D}_{1^{\prime} i}\right)\left(\varepsilon_{\mathrm{L}} \cdot \boldsymbol{D}_{i 1}\right)}{E_{i}-\mathrm{i} \Gamma_{i} / 2-\left(E_{1}+\hbar \omega_{\mathrm{L}}\right)}+\sum_{i} \frac{\left(\varepsilon_{\mathrm{L}} \cdot \boldsymbol{D}_{1^{\prime} i}\right)\left(\varepsilon_{\mathrm{S}}^{*} \cdot \boldsymbol{D}_{i 1}\right)}{E_{i}-\left(E_{1}-\hbar \omega_{\mathrm{L}}\right)}\right|^{2},
$$

where $\boldsymbol{D}_{i j}=\langle i|e \boldsymbol{x}| j\rangle$ is again the dipole matrix element, and $\varepsilon_{\mathrm{s}}$ and $\varepsilon_{\mathrm{L}}$ are the polarization vectors of the emitted photon and the laser photon, respectively. We work within the relativistic dipole approximation in which the replacement $\langle i|\boldsymbol{\alpha} \exp (\mathrm{i} \boldsymbol{k} \cdot \boldsymbol{x})| j\rangle \rightarrow\langle i|\boldsymbol{\alpha}| j\rangle=\mathrm{i}\left(E_{i}-E_{j}\right)\langle i|\boldsymbol{x}| j\rangle$ is made for the transition matrix element. As it will become clear in the sequel, it is necessary to retain $E_{i}$ and $E_{j}$ as relativistic energies, including fine-structure effects. The total cross section is obtained by summing over final angular momentum states of the electron, summing over the polarization states of the emitted photon, and integrating over directions of the emitted photon. If the hydrogen atoms in the initial state are unpolarized, the cross section is also averaged over initial angular momentum states.

The two terms in the sum on the right-hand side correspond to Figs. 1(a) and (b), respectively. If desired, missing prefactors in (3) can be restored according to Eq. (8.7.6) of Ref. [8]. This factor includes a dependence on the laser frequency $\omega_{\mathrm{L}}$ and on the frequency of the emitted photon $\omega_{\mathrm{S}}$ of the form $\omega_{\mathrm{L}} \omega_{\mathrm{s}}^{3}$. In this article, we do not consider frequency shifts related to this additional frequency dependence of the cross section; these effects are already present in the resonance approximation and are included in the basic line shape through a multiplicative factor.

In contrast to the two-photon absorption described by Eq. (2), we now have two terms in the amplitude for the emission-absorption process, which have to be added coherently and correspond to the two diagrams in Fig. 1. In the case of the two-photon absorption described by Eq. (2), the two diagrams analogous to Fig. 1 lead to equivalent contributions, and only one term remains in the matrix element. In Eq. (3), where necessary, the radiative corrections to the energy levels of the intermediate states are included in the denominators, according to the discussion of Ref. [9], and are omitted where they are negligible.

We assume that the laser is tuned through the resonance $|i\rangle=|r\rangle$ near $\hbar \omega_{\mathrm{L}} \approx E_{r}-E_{1}$. The estimate of nonresonant contributions is determined as follows: We define

$$
x=\hbar \omega_{\mathrm{L}}-\left(E_{r}-E_{1}\right)
$$

as the (small) deviation from the resonance energy. The terms with $i=r$ in (3) will give the dominant contribution. All other intermediate states are off resonance. Let $j \neq r$ denote the off-resonant states. Then we have

$$
E_{j}-E_{1}-\hbar \omega_{\mathrm{L}}=E_{j}-E_{r}-x,
$$

and the leading contributions to the cross section are given by

$$
\begin{aligned}
\frac{\mathrm{d} \sigma}{\mathrm{d} \Omega}\left(\varepsilon_{\mathrm{L}}, \varepsilon_{\mathrm{S}}\right) \propto & \frac{\left|A_{r}\left(\varepsilon_{\mathrm{L}}, \varepsilon_{\mathrm{S}}\right)\right|^{2}}{x^{2}+\Gamma_{r}^{2} / 4}+\left|\sum_{j \neq r} \frac{A_{j}\left(\varepsilon_{\mathrm{L}}, \varepsilon_{\mathrm{S}}\right)}{E_{j}-E_{r}-x}\right|^{2}-2 \operatorname{Re}\left\{\left[\frac{A_{r}\left(\varepsilon_{\mathrm{L}}, \varepsilon_{\mathrm{S}}\right)}{x+\mathrm{i} \Gamma_{r} / 2}\right]\right. \\
& \left.\times\left[\sum_{j \neq r} \frac{A_{j}^{*}\left(\varepsilon_{\mathrm{L}}, \varepsilon_{\mathrm{S}}\right)}{E_{j}-E_{r}-x}+\sum_{i} \frac{B_{i}^{*}\left(\varepsilon_{\mathrm{L}}, \varepsilon_{\mathrm{S}}\right)}{E_{i}+E_{r}-2 E_{1}+x}\right]\right\}+\cdots
\end{aligned}
$$

where

$$
A_{i}\left(\varepsilon_{\mathrm{L}}, \varepsilon_{\mathrm{S}}\right)=\sum_{\mu_{i}}\left(\varepsilon_{\mathrm{s}}^{*} \cdot \boldsymbol{D}_{1^{\prime} i}\right)\left(\varepsilon_{\mathrm{L}} \cdot \boldsymbol{D}_{i 1}\right)
$$


and

$$
B_{i}\left(\varepsilon_{\mathrm{L}}, \varepsilon_{\mathrm{S}}\right)=\sum_{\mu_{i}}\left(\varepsilon_{\mathrm{L}} \cdot \boldsymbol{D}_{1^{\prime} i}\right)\left(\varepsilon_{\mathrm{S}}^{*} \cdot \boldsymbol{D}_{i 1}\right)
$$

where $\mu_{i}$ is the angular momentum projection of the state $|i\rangle$. The right-hand side of Eq. (6) contains the dominant resonance in the first term. The second term is only appreciable if the energy level of the state $j$ is separated from the resonance energy level by a fine-structure interval. We neglect the effects of hyperfine splitting in this discussion. The third term is the cross term between the resonant term and the other contributions, which has been examined recently in Ref. [17]. The expression in (6) can be simplified by expanding in powers of $x /\left(E_{j}-E_{r}\right)$ which is effectively an expansion in $\Gamma_{r} /\left(E_{j}-E_{r}\right)$ for $x$ of order $\Gamma_{r}$ or less. Constant terms that contribute a flat background and small corrections proportional to the resonance profile are omitted, with the result

$$
\begin{aligned}
\frac{\mathrm{d} \sigma}{\mathrm{d} \Omega}\left(\varepsilon_{\mathrm{L}}, \varepsilon_{\mathrm{S}}\right) \propto & \frac{\left|A_{r}\left(\varepsilon_{\mathrm{L}}, \varepsilon_{\mathrm{S}}\right)\right|^{2}}{x^{2}+\Gamma_{r}^{2} / 4}+x \sum_{j \neq r} \sum_{k \neq r} \frac{A_{j}\left(\varepsilon_{\mathrm{L}}, \varepsilon_{\mathrm{s}}\right) A_{k}^{*}\left(\varepsilon_{\mathrm{L}}, \varepsilon_{\mathrm{S}}\right)}{\left(E_{j}-E_{r}\right)^{2}\left(E_{k}-E_{r}\right)^{2}}\left(E_{j}+E_{k}-2 E_{r}\right) \\
& -2 x \operatorname{Re}\left\{\frac{A_{r}\left(\varepsilon_{\mathrm{L}}, \varepsilon_{\mathrm{S}}\right)}{x^{2}+\Gamma_{r}^{2} / 4}\left[\sum_{j \neq r} \frac{A_{j}^{*}\left(\varepsilon_{\mathrm{L}}, \varepsilon_{\mathrm{S}}\right)}{E_{j}-E_{r}}+\sum_{i} \frac{B_{i}^{*}\left(\varepsilon_{\mathrm{L}}, \varepsilon_{\mathrm{S}}\right)}{E_{i}+E_{r}-2 E_{1}}\right]\right\}+\cdots .
\end{aligned}
$$

This expression gives a line shape which is a slightly shifted and distorted Lorentz profile, that can be characterized as

$$
\frac{C}{x^{2}+\Gamma_{r}^{2} / 4}+a x+\frac{b x}{x^{2}+\Gamma_{r}^{2} / 4}=\frac{C}{[x-\Delta(x)]^{2}+\Gamma_{r}^{2} / 4},
$$

which, for $x$ of order $\Gamma_{r}$ or less, yields

$$
\Delta(x)=\frac{a}{2 C}\left(x^{2}+\Gamma_{r}^{2} / 4\right)^{2}+\frac{b}{2 C}\left(x^{2}+\Gamma_{r}^{2} / 4\right),
$$

to lowest order in $\Delta(x) / \Gamma_{r}$.

We can now either take the shift of the resonance curve at the half-maximum value as the experimentally observable measure of the apparent shift of the line center

$$
\Delta\left(\frac{\Gamma_{r}}{2}\right)=\frac{a \Gamma_{r}^{4}}{8 C}+\frac{b \Gamma_{r}^{2}}{4 C}
$$

or we can directly investigate the shift of the maximum of the resonance which is

$$
\Delta(0)=\frac{a \Gamma_{r}^{4}}{32 C}+\frac{b \Gamma_{r}^{2}}{8 C}
$$

Both of these formulas are consistent with Low's measure of the asymmetry, and indeed, the latter approach [the investigation of $\Delta(0)$ ] was followed in [9]. Formula (10) provides an estimate of the line-shape distortion away from the maximum.

We assume that $\Gamma_{r}$ is of order $\alpha(Z \alpha)^{4} m c^{2}$, which is the case for hydrogenic $\mathrm{P}$ states, and take into account the fact that the energy differences are of order $(Z \alpha)^{2} m c^{2}$ for states with different principle quantum numbers $n$, or are of order $(Z \alpha)^{4} m c^{2}$ for states with the same principle quantum number. Furthermore, the following order-of-magnitude estimates apply: $C \sim(Z \alpha)^{-4}(e \hbar / m c)^{4}$ (for hydrogenic E1 transitions), $a \sim(Z \alpha)^{-16}\left(m c^{2}\right)^{-3}(e \hbar / m c)^{4}$ [for energy 
numerators and denominators of order $(Z \alpha)^{4} m c^{2}$ ], and $b \sim(Z \alpha)^{-6}\left(m c^{2}\right)^{-1}(e \hbar / m c)^{4}$ [for an energy denominator of order $\left.(Z \alpha)^{2} m c^{2}\right]$. We then we arrive immediately at the order-of-magnitude estimates for $\Delta(0) / \Gamma_{r}$ :

$$
\frac{a \Gamma_{r}^{3}}{32 C} \sim\left(\frac{\Gamma_{r}}{E_{j}-E_{r}}\right)^{3} \sim \begin{cases}\alpha^{3} & \text { for } n_{j}=n_{r} \\ \alpha^{3}(Z \alpha)^{6} & \text { for } n_{j} \neq n_{r}\end{cases}
$$

and

$$
\frac{b \Gamma_{r}}{8 C} \sim \frac{\Gamma_{r}}{E_{j}-E_{r}} \sim \begin{cases}\alpha & \text { for } n_{j}=n_{r} \\ \alpha(Z \alpha)^{2} & \text { for } n_{j} \neq n_{r} .\end{cases}
$$

These order-of-magnitude estimates are not restricted to the particular value $\Delta(0)$; they are valid for the quantity $\Delta(x) / \Gamma_{r}$ for $x$ of the order of $\Gamma_{r}$ or less. In particular, the estimates are relevant for the relative shift of the peak of the resonance in a differential cross section with respect to the natural line width. The contribution proportional to $\alpha$ in the "second term" [see Eq. (15)] vanishes after summing over photon polarizations and angular averaging over the emitted photons, i.e. for the total cross section; however it persists for the differential cross section, as it will be discussed in Sec. 3. In the second term, contributions with $n_{j}=n_{r}$ can therefore be neglected for the total cross section. There are no further cancellations in the total cross section (as compared to its differential form) for the other contributions given in Eqs. (14) and (15).

These estimates can be illuminated by considering the particular example of excitation from the $1 \mathrm{~S}$ state to the $2 \mathrm{P}_{1 / 2}$ state resonance for hydrogen. In the case of a $1 \mathrm{~S}-2 \mathrm{P}_{1 / 2}-1 \mathrm{~S}$ transition, we have for the coefficient $C$ in the first term of Eq. (9) or (10)

$$
C=\frac{1}{2} \int \mathrm{d} \Omega \sum_{\mathrm{s}} \sum_{\mu, \mu^{\prime}}\left|A_{r}\left(\varepsilon_{\mathrm{L}}, \varepsilon_{\mathrm{s}}\right)\right|^{2}=\frac{2^{33} \pi}{3^{22}(Z \alpha)^{4}}\left(\frac{e \hbar}{m c}\right)^{4}+\cdots,
$$

where $\mathrm{d} \Omega$ is the solid angle element of the emitted photon direction, $\mathrm{s}$ is the emitted photon polarization index, $\mu$ and $\mu^{\prime}$ are the angular momentum projection of the initial and final atomic states, and a linearly polarized laser is assumed; the omitted terms indicated by dots are relativistic corrections that are higher-order in $Z \alpha$. For a general outline of the treatment of matrix elements of the type (16), we refer to [18] . The prefactor $1 / 2$ in (16) arises from the average over the initial state total angular momentum projections.

The result is obtained by employing relativistic wave functions. Note that Eq. (16) is different if the electron spin is neglected: in this case, the result should be multiplied by a factor 3 . Specifically, the result obtained with "spinless" Schrödinger wave functions is split in a ratio $1 / 3$ to $2 / 3$ between the $1 \mathrm{~S}-2 \mathrm{P}_{1 / 2}-1 \mathrm{~S}$ and the $1 \mathrm{~S}-2 \mathrm{P}_{3 / 2}-1 \mathrm{~S}$ transitions, according to the different multiplicities of the $2 \mathrm{P}$ states with different angular momentum. Laser spectroscopy can resolve the individual states, so we keep the spin in all intermediate stages of our calculations.

In order to investigate the off-resonant frequency shifts, we turn our attention to the "first term" given in Eq. (14). Its existence has been pointed out in [19]. Only terms for which both $j$ and $k$ correspond to the $2 \mathrm{P}_{3 / 2}$ state make an appreciable contribution. The coefficient $a$ is given by

$$
a=\int \mathrm{d} \Omega \sum_{\mathrm{s}} \sum_{\mu, \mu^{\prime}} \frac{\left|A_{j}\left(\varepsilon_{\mathrm{L}}, \varepsilon_{\mathrm{s}}\right)\right|^{2}}{\left(E_{j}-E_{r}\right)^{3}}=\frac{2^{50} \pi}{3^{22}(Z \alpha)^{16}\left(m c^{2}\right)^{3}}\left(\frac{e \hbar}{m c}\right)^{4}+\cdots .
$$

\footnotetext{
${ }^{4}$ In this reference, the problem of electric field induced decay of the $2 \mathrm{~S}$ state is examined within the QED scattering formulation. The formalism is very similar to the present considerations, because the electric field acts as a zero frequency photon, which is very far from resonance.
} 
We use the well-known result

$$
\Gamma_{2 \mathrm{P}_{1 / 2}}=\frac{2^{8}}{3^{8}} \alpha(Z \alpha)^{4} m c^{2}+\cdots,
$$

which, together with Eqs. (16) and (17), yields

$$
\frac{a \Gamma_{r}^{3}}{32 C}=\frac{2^{36}}{3^{24}} \alpha^{3}+\cdots \approx 9.5 \times 10^{-8},
$$

corresponding to a frequency shift of about $9.5 \mathrm{~Hz}$.

The "second term" given in Eq. (15) has been investigated in Refs. [17, 19] We obtain for this contribution $\left(b=b_{1}+b_{2}\right)$

$$
\begin{aligned}
& b_{1}=-\int \mathrm{d} \Omega \sum_{\mathrm{s}} \sum_{\mu, \mu^{\prime}} \operatorname{Re} A_{r}\left(\varepsilon_{\mathrm{L}}, \varepsilon_{\mathrm{S}}\right) \sum_{j \neq r} \frac{A_{j}^{*}\left(\varepsilon_{\mathrm{L}}, \varepsilon_{\mathrm{S}}\right)}{E_{j}-E_{r}}=-\frac{9.3 \pi}{(Z \alpha)^{6} m c^{2}}\left(\frac{e \hbar}{m c}\right)^{4}+\cdots \\
& b_{2}=-\int \mathrm{d} \Omega \sum_{\mathrm{s}} \sum_{\mu, \mu^{\prime}} \operatorname{Re} A_{r}\left(\varepsilon_{\mathrm{L}}, \varepsilon_{\mathrm{S}}\right) \sum_{i} \frac{B_{i}^{*}\left(\varepsilon_{\mathrm{L}}, \varepsilon_{\mathrm{S}}\right)}{E_{i}+E_{r}-2 E_{1}}=-\frac{3.6 \pi}{(Z \alpha)^{6} m c^{2}}\left(\frac{e \hbar}{m c}\right)^{4}+\cdots
\end{aligned}
$$

This yields for $Z=1$,

$$
\frac{b \Gamma_{r}}{8 C}=-7.4 \times 10^{-2} \alpha(Z \alpha)^{2}+\cdots \approx-2.9 \times 10^{-8},
$$

corresponding to a frequency shift of $-2.9 \mathrm{~Hz}$. Interference terms between the $2 \mathrm{P}_{1 / 2}$ and $2 \mathrm{P}_{3 / 2}$ states vanish for $b_{1}$, but make an appreciable contribution to $b_{2}$.

In total, the frequency shift $\Delta(0)$ for the $1 \mathrm{~S}-2 \mathrm{P}_{1 / 2}-1 \mathrm{~S}$ transition is

$$
\Delta(0)=(9.5-2.9) \mathrm{Hz}=+6.6 \mathrm{~Hz} \quad\left(\text { for } 1 \mathrm{~S}-2 \mathrm{P}_{1 / 2}-1 \mathrm{~S}\right) .
$$

This would be very difficult to detect in comparison to the natural line width of about $100 \mathrm{MHz}$. We now turn our attention to the four-photon process shown in Fig. 2. From the usual $S$-matrix formalism we infer the cross-section to be proportional to

$$
\begin{gathered}
\frac{\mathrm{d} \sigma}{\mathrm{d} \Omega} \propto \\
\left|\sum_{i j k} \frac{\left(\varepsilon_{t}^{*} \cdot \boldsymbol{D}_{1 k}\right)\left(\varepsilon_{\mathrm{s}}^{*} \cdot \boldsymbol{D}_{k j}\right)\left(\varepsilon_{\mathrm{L}} \cdot \boldsymbol{D}_{j i}\right)\left(\varepsilon_{\mathrm{L}} \cdot \boldsymbol{D}_{i 1}\right)}{\left[E_{k}-\left(E_{1}+2 \hbar \omega_{\mathrm{L}}-\hbar \omega_{\mathrm{s}}\right)\right]\left[\left(E_{j}-\frac{i}{2} \Gamma_{j}\right)-\left(E_{1}+2 \hbar \omega_{\mathrm{L}}\right)\right]\left[E_{i}-\left(E_{1}+\hbar \omega_{\mathrm{L}}\right)\right]}+\ldots\right|^{2} .
\end{gathered}
$$

In this case, the energies of the two emitted photons $\omega_{\mathrm{s}}$ and $\omega_{\mathrm{t}}$ are subject to the condition $\omega_{\mathrm{s}}+\omega_{\mathrm{t}}=2 \omega_{\mathrm{L}}$. An integration over one of the energies is required; this has no influence on our considerations below. In this context, it is probably worthwhile to note that similar calculations involving four-photon processes have to be performed in the context of third-harmonic generation (see [8]). Terms left out in the expression (24), denoted by “...", correspond to the different timeorderings of photon emission and interactions with the laser [cf. Eq. (2)]. In the experiment, the laser is tuned through the two-photon resonance so that $E_{1}+2 \hbar \omega_{\mathrm{L}} \approx E_{r}$ where $E_{r}$ is the energy of the $2 \mathrm{~S}$ state. In the cross section (24), it is only the denominator with summation variable $j$

\footnotetext{
${ }^{5} \mathrm{In}[17$, a result of $4.89 \mathrm{~Hz}$ is reported for this term.

${ }^{6} \mathrm{In}$ [17], a result of $+2.9 \mathrm{~Hz}$ is reported for this term, whereas in 19], the authors give an additional factor of $4 / 9$ and a sign change to obtain a result of $-1.3 \mathrm{~Hz}$. We agree with the magnitude of the former result and the sign of the latter result. Note that the term referred to as $\delta_{1 \mathrm{~S}, 1 \mathrm{~S}}^{2 \mathrm{P}}$ in] corresponds to $\Delta(0)$ in our notation.
} 
which may become resonant, and which therefore is in need of a modification $E_{j} \rightarrow E_{j}-\frac{\mathrm{i}}{2} \Gamma_{j}$. All other denominators in (24) remain off-resonant.

For a Doppler-free 1S-2S two-photon process with the absorption of two counter-propagating laser photons, a typical off-resonant contribution is given by the case when $|j\rangle$ equals the $3 \mathrm{~S}$ or $4 \mathrm{~S}$ (in general, $n \mathrm{~S}$ ) state. The matrix elements in the numerator of (24) have the same orderof-magnitude for both the resonant and the off-resonant cases, in analogy to the $C_{j r}$ being of order unity for the two-photon process described by Eq. (6).

We can now use exactly the same formalism as was used in the analysis of the two-photon cross section (3). A calculation shows that the nonresonant contributions to the four-photon process described by Eq. (24) result in a shift of the peak of the cross section by

$$
\delta \omega_{2 L} \sim \frac{\Gamma_{r}^{2} / \hbar}{E_{j}-E_{r}}
$$

where $E_{j}$ denotes the energy of a typical nonresonant state. The index $2 L$ indicates that there are two absorbed laser photons.

We will now consider the $1 \mathrm{~S}-2 \mathrm{~S}$ two-photon transition in atomic hydrogen. The imaginary part of the energy ("the width") of the $2 \mathrm{~S}$ state is given to within a good approximation by the two-photon radiative lifetime which is of the order of [24]

$$
\Gamma_{2 \mathrm{~S}} \sim \alpha^{2}(Z \alpha)^{6} m c^{2} .
$$

This order-of-magnitude estimate can also be inferred by considering the poles and corresponding residues of the low-energy part of the two-photon self-energy, as expressed in nonrelativistic quantum electrodynamics [the relevant formula is given in Eq. (16) of [25]]. In view of Eqs. (25) and (26),

$$
\delta \omega_{2 L} \sim \alpha^{4}(Z \alpha)^{10} \frac{m c^{2}}{\hbar} .
$$

This is a factor of $\alpha^{2}(Z \alpha)^{4}$ smaller than the width of the $2 \mathrm{~S}$ state. For the $1 \mathrm{~S}-2 \mathrm{~S}$ transition in atomic hydrogen, this estimate means that the off-resonant contributions will enter at the level of $10^{-14} \mathrm{~Hz}$. This is far smaller than the natural width of the $2 \mathrm{~S}$ state and irrelevant for current measurements which have an experimental uncertainty of about $46 \mathrm{~Hz}$ [1].

In many cases, the shift of the peak of a resonance by off-resonant contributions is much smaller than the natural width of the resonance itself. This is not surprising and can be understood qualitatively. A careful inspection of the physics associated with Eq. (9) is sufficient. The derivative of the narrow resonance contribution [first term of Eq. (9)] near $x=0$ changes rapidly in the vicinity of the resonance; by contrast, the off-resonant contributions [the remaining terms of Eq. (9)] are rather flat in that frequency region. The maximum of the shifted resonance profile must fulfill the condition that the sum of the derivatives of resonant and off-resonant contributions must add up to zero. The derivative of the resonant contribution changes rapidly, even within its natural width, and compensates that of the off-resonant contribution in the vicinity of the original resonant contribution. This implies that the shifted peak of the resonance must lie very close to its original value and can only be shifted by a frequency difference which is much smaller than the natural width of the initial and final states in the resonance transition.

Specifically, for the hydrogenic $1 \mathrm{~S}-2 \mathrm{~S}$ transitions, there may be further nonresonant contributions, for example those involving the absorption of three laser photons, leading to an excitation of the $2 \mathrm{~S}$ level into a $\mathrm{P}$ state of the continuum with energy $E_{2 \mathrm{~S}}+\hbar \omega_{\mathrm{L}}$. This further nonresonant effect will be proportional to the intensity of the laser beam. Because the transition matrix elements for bound state-continuum transition are enhanced by a factor $(Z \alpha)^{-3 / 2}$ as compared to 
transitions between two bound states, this effect will be of the order of $\alpha^{4}(Z \alpha)^{7}$. However, the order-of-magnitude of this effect is still much smaller than the natural width of the transition, in agreement with the qualitative discussion in the preceding paragraph.

Radiative vertex corrections to the photon scattering also contribute to the line shape (see the discussion in [9] and Fig. 3). So, our result in Eq. (27) is not meant to indicate that the peak of the resonance corresponds to the $1 \mathrm{~S}-2 \mathrm{~S}$ energy level difference at the level of precision of $\delta \omega_{2 L}$, but it indicates that possibly interesting off-resonance effects due to nearby atomic bound states are negligible for the two-photon high-precision experiments.

We conclude this section by noting that there is a further nonresonant contribution originating from the possibility of the nonrelativistically forbidden, nonresonant one-photon magnetic $1 \mathrm{~S}-2 \mathrm{~S}$ transition. This contribution is, however, Doppler-broadened [cf. Eq. (2)] and is therefore negligible to a very good approximation. Its magnitude will depend on the experimental conditions. Also, it is known that the second-order Doppler effect and the AC Stark shifts in the intense laser field are the most important systematic effects in current experiments [1] (these necessitate an extrapolation to zero laser field).

\section{Differential measurement}

We consider the process $1 \mathrm{~S}-2 \mathrm{P}_{3 / 2}-1 \mathrm{~S}$ described by the cross section (3). There is a nearby $2 \mathrm{P}_{1 / 2}$ level which is separated from the $2 \mathrm{P}_{3 / 2}$-level by the fine-structure interval

$$
E\left(2 \mathrm{P}_{3 / 2}\right)-E\left(2 \mathrm{P}_{1 / 2}\right)=\frac{(Z \alpha)^{4}}{32} m c^{2}
$$

This is two orders of $(Z \alpha)$ smaller than the hydrogenic energy difference between states with different quantum numbers, which is of order $(Z \alpha)^{2}$. According to Eq. (15), the nearby $2 \mathrm{P}_{3 / 2}$ level should lead to a large nonresonant contribution of order

$$
\delta \omega\left(1 \mathrm{~S} \rightarrow 2 \mathrm{P}_{3 / 2} \rightarrow 1 \mathrm{~S}\right) \sim \frac{\left(\Gamma_{2 \mathrm{P}_{1 / 2}}\right)^{2} / \hbar}{E\left(2 \mathrm{P}_{3 / 2}\right)-E\left(2 \mathrm{P}_{1 / 2}\right)} \sim \alpha^{2} \frac{(Z \alpha)^{8}}{(Z \alpha)^{4}} \frac{m c^{2}}{\hbar}=\alpha^{2}(Z \alpha)^{4} \frac{m c^{2}}{\hbar}
$$

which is two orders of $(Z \alpha)^{2}$ larger than the result in Eq. (23). Based on Eqs. (15), (18) and (20), we obtain for the nonresonant frequency shift of the process $1 \mathrm{~S}-2 \mathrm{P}_{3 / 2}-1 \mathrm{~S}$ the result

$$
\delta \omega\left(1 \mathrm{~S} \rightarrow 2 \mathrm{P}_{3 / 2} \rightarrow 1 \mathrm{~S}\right)=K\left[2^{3}\left(\frac{2}{3}\right)^{16} \alpha^{2}(Z \alpha)^{4} \frac{m c^{2}}{\hbar}\right]
$$

where $K$ is a numerical factor which depends on spin and photon polarizations. When summing over the final-state spin polarizations of the electron, summing over the photon polarizations and integrating over the angle of the emitted photon, $K$ vanishes. In this case, the dominant off-resonant frequency is of order $\alpha^{2}(Z \alpha)^{6} m c^{2} / \hbar$ [see Eq. (11) and Refs. [9,17]]. However, the coefficient $K$ does not vanish when spin-polarized hydrogen is used and measured in the experiment, or differential cross sections are considered.

\footnotetext{
${ }^{7}$ Some arguments presented in the seminal paper 9] with regard to nonresonant levels which are "removed from the (intermediate state) $m$ (of the two-photon process) by a fine- or hyperfine-structure splitting" do not appear to be universally applicable [it was argued that the smaller energy denominator in this case is compensated by smaller transition matrix elements $\boldsymbol{D}_{m 1}$ in the numerator of (3)]. This argument is certainly applicable to offresonant states which lie very close to the initial state of the two-photon process. However, in the presence of an intermediate state very close to the resonance state, whose transition matrix elements are of the same order as for the resonant state, this argument does not appear to hold universally. In this case, a treatment including fine structure effects cannot be avoided, especially if differential cross sections are considered.
} 
For the transition $1 \mathrm{~S}(m=1 / 2)-2 \mathrm{P}_{3 / 2}-1 \mathrm{~S}(m=1 / 2)$, there is a nonvanishing interference term which depends on the polarization of the laser beam (here, $m$ refers to the projection of the electron spin onto the $z$ axis). Specifically, $K=1 / 2$ for linearly polarized light along the $z$ axis, and $K=2$ for $\sigma^{-}$-polarized light. Here, $\sigma^{-}$-polarized light is to be understood as circularly polarized light with a polarization vector in the $x-y$ plane; this polarization being chosen such that the light can drive both transitions $1 \mathrm{~S}(m=1 / 2)-2 \mathrm{P}_{3 / 2}$ and $1 \mathrm{~S}(m=1 / 2)-2 \mathrm{P}_{1 / 2}$. For $\sigma^{+}$ polarized light, which can drive a transitions $1 \mathrm{~S}(m=1 / 2)-2 \mathrm{P}_{3 / 2}$ but cannot drive $1 \mathrm{~S}(m=1 / 2)-$ $2 \mathrm{P}_{1 / 2}, K$ again vanishes. The average $\bar{K}=5 / 4$ for the two experimental set-ups for which $K$ is nonvanishing corresponds to a shift of order $\delta \nu=\delta \omega /(2 \pi)=0.28 \mathrm{MHz}$. This could be within the range of current measurements, even though it has to be compared to the natural width of the hydrogenic $2 \mathrm{P}$ states which is of the order of $\Gamma(2 \mathrm{P})=100 \mathrm{MHz}$.

We also note that $K$ is nonvanishing if the experiment is restricted to the measurement of specific photon polarizations. For example, for the case of an initial $1 \mathrm{~S}(m=1 / 2)$ state, with a laser beam polarized along the $z$ axis, and emitted photon polarizations along either the $x$ or the $y$ axis, we obtain $K=-1$, corresponding to $\delta \omega /(2 \pi)=-0.23 \mathrm{MHz}$. Also, with a both the laser beam and the emitted photons polarized along the $z$ axis, we obtain $K=1 / 2$, corresponding to $\delta \omega /(2 \pi)=+0.11 \mathrm{MHz}$.

The obvious idea would be to switch "on or off" the nonresonant contribution from the nearby $2 \mathrm{P}_{1 / 2}$ state. This can be reached by switching from a measurement involving unpolarized light and/or spin-unpolarized hydrogen to a measurement with polarized light and/or spin-polarized atomic hydrogen, as described above.

\section{Conclusions}

We have analyzed nonresonant contributions to two-photon and four-photon processes shown in Figs. 11 and 2. We provide a formula for the distorted Lorentz profile in Eq. (10). For the hydrogenic $1 \mathrm{~S}-2 \mathrm{P}_{1 / 2}$ transitions, the result for the off-resonant frequency shifts is given in Eq. (23).

For the hydrogenic 1S-2S transition, we obtain the order-of-magnitude estimate (25) for the nonresonant corrections to the observed transition frequency as one of the main results of this paper. The nonresonant effect due to off-resonant atomic bound states is given in Eq. (27) and is negligible on the level of any conceivable measurement (see Sec. 2). Notably, this nonresonant correction is orders of magnitude smaller than other systematic effects like the second-order Doppler effect and the AC Stark shift. However, three-photon transitions to the continuum and relativistic and radiative corrections to the two-photon process (a typical diagram is given in Fig. 3) will dominate the off-resonant frequency shifts for two-photon transitions (see the discussion in Sec. 2), and these and other effects can be orders of magnitude larger than the effect due to off-resonant S levels given in Eq. (27) (see also the related discussion in [19]). While the magnitude of these effects depends on the particular experimental conditions (e.g., the laser intensity or the measurement of differential vs. total cross sections), we conclude that the natural line shape of the two-photon process will remain Lorentzian to a very good approximation, enabling the comparison of theory and experiment for the $1 \mathrm{~S}-2 \mathrm{~S}$ transition to well below the $1.3 \mathrm{~Hz}$ natural line width.

We discuss the possibility of a differential measurement by which a nonresonant contribution from a nearby state could be detected in current Lamb shift measurements (see Sec. 3). For the specific experimental setup outlined in Sec. 3, the requirement would be to determine the line center - defined as the frequency corresponding to the maximum cross section - to within a relative uncertainty of the order of one part in 400; this measurement could be feasible with the current technology. The nonresonant shift in this case is indicated in Eq. (30). Clearly, an 
enhancement of nonresonant contributions can be expected in an atomic system where two levels with equal quantum numbers are lying very close to each other.

The discussion in Sec. 3 shows that nonresonant levels which lie close to a resonance may result in surprisingly large shifts of the peaks of the cross sections. However, the qualitative considerations presented near the end of Sec. 2 always remain valid: the "nonresonant shift" $\delta \omega$ constitutes only a fraction of the natural radiative width of the resonance if the natural width of the transition is small compared to the energy interval between the resonant and the nearest off-resonant state. This consideration also applies if levels lie very close to each other, and the denominator in Eq. (25) or (29) becomes very small: one example would be hyperfine-singlet and hyperfine-triplet hydrogenic $\mathrm{S}$ levels $(F=0,1)$ which are separated from each other only by the hyperfine structure splitting.

\section{Acknowledgements}

UDJ is grateful for continued support extended by NIST during a series of research appointments, and to D. Kelleher, K. Pachucki and V. M. Shabaev for helpful discussions.

\section{References}

[1] M. Niering, R. Holzwarth, J. Reichert, P. Pokasov, T. Udem, M. Weitz, T. W. Hänsch, P. Lemonde, G. Santarelli, M. Abgrall, P. Laurent, C. Salomon, and A. Clairon, Phys. Rev. Lett. 84, 5496 (2000).

[2] U. D. Jentschura, P. J. Mohr, and G. Soff, Phys. Rev. Lett. 82, 53 (1999).

[3] K. Melnikov and T. v. Ritbergen, Phys. Rev. Lett. 84, 1673 (2000).

[4] V. A. Yerokhin, Phys. Rev. A 62, 012508 (2000).

[5] U. D. Jentschura, P. J. Mohr, and G. Soff, Phys. Rev. A 64, 042512 (2001).

[6] P. J. Mohr and B. N. Taylor, Rev. Mod. Phys. 72, 351 (2000).

[7] W. Kramers and W. H. Heisenberg, Z. Phys. 31, 681 (1925).

[8] R. Loudon, The Quantum Theory of Light, third ed. (Oxford University Press, 2000).

[9] F. Low, Phys. Rev. 88, 53 (1952).

[10] C. Billionet, J. Phys. I (France) 5, 949 (1995).

[11] S. R. Lundeen, PhD thesis, Harvard University, 1981 (unpublished).

[12] L. N. Labzowsky, Zh. Éksp. Teor. Fiz. 85, 869 (1983).

[13] L. N. Labzowsky, G. Klimchitskaya, and Y. Dmitiriev, Relativistic Effects in the Spectra of Atomic Systems (IoP, Bristol, 1993).

[14] V. V. Karasiev, L. N. Labzowsky, A. V. Nefiodov, V. G. Gorshkov, and A. A. Sultanaev, Phys. Scr. 46, 225 (1992).

[15] L. N. Labzowsky, V. V. Karasiev, I. Lindgren, H. Persson, and S. Salomonson, Phys. Scr. T 46, 150 (1993). 
[16] L. N. Labzowsky, V. V. Karasiev, and I. A. Goidenko, J. Phys. B 27, L439 (1994).

[17] L. N. Labzowsky, D. A. Solovyev, G. Plunien, and G. Soff, Phys. Rev. Lett. 87, 143003 (2001).

[18] M. Hillery and P. J. Mohr, Phys. Rev. A 21, 24 (1980).

[19] L. N. Labzowsky, D. A. Solovyev, G. Plunien, and G. Soff, e-print physics/0201067, version 1 of 30 Jan, 2002.

[20] U. D. Jentschura, G. Soff, and P. J. Mohr, Phys. Rev. A 56, 1739 (1997).

[21] M. Göppert-Mayer, Ann. Phys. (N. Y.) 9, 273 (1931).

[22] W. Kaiser and C. G. Garret, Phys. Rev. Lett. 7, 229 (1961).

[23] W. Demtröder, Laserspektroskopie, 3 ed. (Springer, Berlin, 1993).

[24] R. Marrus and P. J. Mohr, Adv. At. Mol. Phys. 14, 181 (1978).

[25] K. Pachucki, Phys. Rev. A 63, 042503 (2001). 